\title{
The Theoretical Principles of the
} Body-Centered Therapy to Promote Affective Attunement in Children with Autism Spectrum Disorder

\author{
Magda Di Renzo \\ Institute of Ortofonologia (IdO), Rome, Italy \\ Email:m.direnzo@ortofonologia.it
}

How to cite this paper: Di Renzo, M. (2017) The Theoretical Principles of the Body-Centered Therapy to Promote Affective Attunement in Children with Autism Spectrum Disorder. Journal of Behavioral and Brain Science, 7, 545-556. https://doi.org/10.4236/jbbs.2017.712039

Received: September 26, 2017 Accepted: November 19, 2017 Published: November 22, 2017

Copyright $\odot 2017$ by author and Scientific Research Publishing Inc. This work is licensed under the Creative Commons Attribution International License (CC BY 4.0).

http://creativecommons.org/licenses/by/4.0/

\begin{abstract}
Background: The roots of autism spectrum disorders become evident in first attunement mechanisms between mother and child that allow a first level of mentalizing. Neurosciences and current developmental theories confirm the existence of defensive mechanisms related to body and affectivity that psychodynamic theories had already highlighted. Reading child's behavior not only by administering tests but also through careful clinical observations allows a better understanding of the communication and social difficulties present in autistic children. The identification of the zone of proximal development can promote a therapeutic intervention that respects the individuality of the child and the specificity of his relational approach to the world. Conclusion: The paper presents the theoretical principles of a bodycentered therapy to promote the attunement processes necessary to activate cognitive resources.
\end{abstract}

\section{Keywords}

Autism Spectrum Disorders, Body-Centered Therapy, Affective Attunement, Mentalizing, Clinical Observation

\section{Introduction}

As it is well known, a number of changes were made to pervasive developmental disorders (PDDs) in the recently released Diagnostic and Statistical Manual of Mental Disorders, 5th edition [1]. The proposed DSM-5 criteria for autism spectrum disorders have been reduced so that the ASD symptoms are best represented 
in a two-domain model of social-communication deficits and restricted and repetitive interests/behaviors (RRB). Aiming towards a dimensional approach, the DSM-5 emphasizes the concept of spectrum to reflect a continuum that defines more accurate and descriptive specifiers (Severity level; Co-existing conditions and genetic or medical diagnoses; Intellectual functioning; Language level) that need a thorough clinical evaluation. The definition of diagnostic specifiers, in fact, requires the professional, in addition to the use of standardized tests, even a good expertise in collecting clinical and anamnestic data for being integrated with the direct observation of the child. The latter, in fact, can never be replaced by the exclusive use of scores obtained in the assessment process.

It seems clear, in this perspective, the competence that the clinician must have regard to development and its facets. Otherwise, the risk is to define a diagnostic framework only for the presence of symptomatic behaviors, without considering a causal elaboration for each behavior, without highlighting the potential present and without a precise definition of the areas that need intervention in terms of priority in the genesis of the disorder.

The observation must then consider the different ways in which the autistic child expresses his atypia in cognitive, language, behavioral and emotional areas, to be able to place the therapeutic intervention in the zone of proximal development indicated by Vygotskij [2].

In this perspective, the developmental approach, which takes into account all the components that come into play during growth, can provide the right tools to deal with the diagnostic and therapeutic process with full respect for the individuality of each child. The developmental model gives importance to the child's motivation to learn and the naturalness of the context in facilitating those normal tuning processes that form the basis of relationships and therefore of communication [3]-[15].

\section{Main Context}

This article aims to highlight the importance of adopting a developmental approach which defines the zone of proximal development so considering the body dimension as an essential basis to activate the resources of the child and help him in that attunement process he lacks. This could stimulate a motivating communication in the child.

The latest developmental and neuroscientific researches show that knowledge is the result of a process that starts from the bodily-affective area to arrive slowly, and through required steps, to the mental dimension.

Apart from ethiopathogenesis, what we can find in the autistic child is an arrest in the attunement development that determines a serious failure in the imitation processes, a distortion in sensory and emotional processing and, as a result, a deficit in the cognitive dimension.

The authors of psychodynamic approach, interested in the phenomenology of the disorder, showed different defensive mechanisms that all refer to a peculiar 
way of experiencing and living the body dimension.

Frances Tustin pointed out that when the autistic child experiences the separation from the mother before he has developed the transitional activity described by Winnicott, he starts to generate pathological auto-sensuous reactions instead of expected fantasy, imagination, thought and play [16] [17] [18] [19] [20].

In the typical early childhood development autistic objects are normal and gradually give way to the creation of fantasies and illusions and finally to the thought. In autistic children who instead do not accept the separateness from the mother, these objects are manipulated in a stereotypical, over-materialized, only bodily way because these children have never learned to "tolerate" the world as it is and then to adjust to it. Autistic sensation objects [17] are, therefore, material objects, "protections" (used in place of the parents and not to evoke their absence) and are opposed to the development of thought.

The auto-sensuous reactions, as opposed to what normally happens in the early stages of a child's life, do not allow to define a bodily boundary because they are kept alive to divert the attention of the autistic child from everyday experiences. Autistic sensation shapes [18], acting as bodily traces of previous pleasant experiences, allow the child to console and defend himself from the sense of loss or abuse of the outside world stimuli.

In recent years Smith [21], with his empathy imbalance hypothesis, argued that autistic behaviors and stereotypic patterns are defenses the children with autism develop to manage the excess of feelings and emotions that are not addressed in the relationship. In this view, the autistic behavior is an adaptive response to empathic imbalance. Avoidance behavior, obsessive interests, and insistence on routines may regulate the stress that stems from living among people whose behavior is difficult to comprehend but whose emotions are all too readily sensed.

Donald Meltzer [22] described the defensive mechanisms related to the body through the concepts of sensory dismantling and adhesive identification stressing that the autistic child has a form of primitive permeability which makes him extremely vulnerable to the outside world. According to the author, autistic children have a particular speed in mental processes that makes them prone to perceive stimuli from the outside world as a sort of bombardment.

Through the dismantling, autistic children fragment the experience so to no longer perceive it as dangerous because this form of segmentation makes impossible any form of integration. Since the dismantling is a defensive mechanism earlier than the splitting, it allows the parceling of the experience which occurs through the passive attitude of the child and that constantly diverts him from the relationship with the object. Thanks to the underlying mechanism that is the adhesive identification, the child is able to adhere completely to the surface of objects avoiding to perceive the three-dimensionality which gives them depth, so eluding the experience of limit that anticipates the sense of separateness.

The adhesive identification also provides an explanation of the preference that 
autistic children have for the proximal senses than distal.

The sense of touch, for example, promotes the experience of merging, the baby seeks, whereas hearing or sight confront him with that distance that he would cancel and that are thus weakened. Fordham [23], considers the autism as a disorder of the Self, as a distorted state of integration, owing its persistence to failure of the Self to deintegrate. Fordham proposed the term deintegration as a property of the Self behind the ego formation. The Self deintegration is therefore understood as a normal stage of development in which the child is able to recognize objects as different from the Self (through the physiological process of integration and deintegration that allows the differentiation of the original psychosomatic unity). Reconnecting to immunological researches that discovered the existence of defensive reactions carried out to cancel or reject the not self-objects, Fordham assumed that only the objects assimilable from the Self can promote vital biochemical processes and, in the case of autism, a functional disintegration occurs to hold off the not self-objects, that is a kind of auto-immune disease that prevents vital processes.

Damasio [24], more recently, showed that the Self is built in stages, is constructed starting from the meeting of the body with the outside world objects, according to primordial feelings. These are spontaneous reflections of the state of the living body which form the basis of cognitive processes. The Self comes to mind from the sensations belonging to the brain stem, which represents, in his opinion, not a mere conduit for signals, but a first decisional location, a center of information processing. The protoself is an integrated collection of separate neural patterns that map, moment by moment, the most stable aspects of the organism's physical structure, it is a sort of deep root that cannot be alienated. The knowledge that makes conscious our mind must be built according to a bottom-up mode, from body to brain. The conscious mind emerges from a link between the body and the object of knowledge. Primordial feelings, produced by the protoself, precede all other feelings and uniquely refer to the living body that is interconnected with its specific brain stem.

According to Damasio [25], in brief, in the cognitive process we can distinguish the following steps: 1) an object involves the body when it is looked at, touched or heard from a specific perspective, 2) the involvement induces a change in the body, 3) the object's presence is felt, 4) the object has been made prominent, 5) the object becomes part of the child's knowledge and settles in the memory. The Self, therefore, comes to mind in the form of images that narrate, without interruption, the story of these entanglements.

What these authors have shown, in physiological or pathological sense, it is the centrality of the body in child development, hypothesis also supported by Stern's studies about inter-subjectivity which highlighted the importance of the affective attunement. The mechanisms underlying it are the amodal properties of experience (intensity, time, shape, movement and number) that allow the interchangeability of sensory modes for the different behavioral expressions. What 
characterizes the attunement, distinguishing it from other mechanisms, is its ability to respond not only to affective categories but also to vital affections such as the increasing and decreasing of excitement. This possibility guarantees the continuity of the mother-child experience and allows, in the developmental process, to experience the vital affections in the form of dynamic changes in ourselves or in others.

The affective attunement assumes the presence of imitation and contagion which both express an exclusive interest in overt behavior and which are both interfered in the autistic children. The child, according to this perspective, is not able to get the mirroring nor can respond to the variations on a theme that mother provides him [9] (Stern 1985) because the imitation, already lacking, cannot favor an adequate affective attunement. Gaddini [26] highlighted the fact that in the first weeks of life the child perceives through bodily modifications respect to the object and that the differentiation of perception and memory systems starts from this early communion of perception and physical imitation. What Gaddini stressed so bright is the fact that the first manifestations of the imitation process have to do with being and not with having, so that the imitative conducts can be considered as precursors of the ego adaptation processes.

In this perspective, the autistic disorder consists of a lack or a significant deficiency in the imitation processes that can be defined as a difficulty to pass from the stage of imitate to perceive to the stage of imitate to be. This is a child's difficulty in getting hold, in the sense of Winnicott [27] [28] [29] from the emotional dimension of the mother which is predominantly expressed by bodily dimension through the modulation of muscle tone intended as an essential element for the transition to the imitate to be.

The tonic dialogue [30] between mother and child, as well as forming the basis for the future development of empathy, also allows the construction of a plot for the semantic processing of the contents carried in the relationship. Without the hug that holds and that allows the child to recognize himself as a separate individual there can be no exploration in the world nor the ability to respond to the other.

More recently Reddy [13], commenting on the imitation studies of Nadel [12], emphasized the interpersonal dimension already present from the first days of life with regard to imitation, showing that emotional involvement is essential also for the repetition of those simple gestures, such as tongue protrusion, that before were seen only through an intra-individual perspective. Many authors confirmed this hypothesis by moving more and more attention also to the gestational period as the beginning of possible attunement, so that Trevarthen [5] coined the term sinus rythm to refer to the existing dialogue between mother and baby during pregnancy.

Neuroscience highlighted the fact that the inter-subjective characteristic of first experiences, as well as outlined until now, in the brain is sustained by the presence of mirror neurons. 
Through the embodied simulation [31] it is possible to experience and then understand the tactile and painful experience of other people simply observing them, because even the only others' observation activates the same neural circuits that are responsible for others' feeling. The word simulation is to be understood as an attempt to mimic the characteristics of a process in order to assimilate its essence and is expressed as a modeling mechanism. In this sense it differs from the process proposed by the "simulation theory" (as a result of a deliberate act of will) because it is characterized as an automatic process, not conscious and predeclarative.

The classical cognitive approach, which expresses the vision of a fully disembodied human mind, in the sense that the ability to understand others' intentions would simply be due to met are presentations, appears, in light of this, simplistic and inadequate to the complexity of the situation [32]. Through the mechanism of embodied simulation, which enables two different bodies to share a functional state, the "objectual other" becomes "another self" and thanks to this it is possible to build the foundations for social intelligence. In this sense the body, simultaneously perceived as a place of subjective and objective experience, allows the structuring of that perception of the personality experience that can be attributed also to the others. The same sensory-motor circuits that control body movements and perception, in fact, give structure to abstract thought too [33] [34]. This identity relation, which is transversal to all the forms of interpersonal relationships, is defined by Gallese [32] as shared manifold hypothesis and promotes, as already underlined, the intersubjectivity and the attribution of intentionality to the others. The impossibility of building a we-centric space, as a basis for establishing a connection with the intentional relations of others, does not allow the development of a resonance able to favor anticipations of the behavior of others. Experimental data on postural attitudes show that autistic children are not able to use feed-forward mechanisms but essentially use the data coming from the sensory periphery, unable to adopt anticipatory postural attitudes. Experiments with high-functioning autistic subjects showed that recognition and imitation of other people's emotions occurs through different strategies from those used by normal subjects (there is an absence of the premotor mirror circuit, underactivation of insula and amygdala and a hyperactivity of the visual cortex). The lack of embodied simulation does not allow, in this case, to give an experiential content to the emotions of others and access to the other would remain limited to the cognitive interpretation of sensory conveyed by an external event.

In the Italian developmental approach called Turtle Project [35] the focus of the intervention is constituted from the body's relationship with the child that also involves parents in individual and/or group settings. The fundamental objective is to help parents to interpret the atypia of the child for creating a shared care that could form the basis for a first form of communication. Meet the need of the child, as mothers know usually do, is quite different from helping the child to mentalizing his need. To make this happen it is necessary that adult does 
not refuse the child's dysfunctional behaviors, as occurs with the use of behavior modification techniques, but that accept and transform them to kick off that kind of dance [9] which allows to bring within the context variations on a theme. Only starting from such tuning can be activate the imitation mechanism that allows the child to imitate to be rather than imitate to perceive.

The reverie capacity [36] [37] of the mother may in fact be reactivated if the therapist is able to make interpretable for her the child's behavior and, only in this case, the mother can start to put in place that hypothesis of semantic nature [38] [39] [40] which founds other's mind. The communication starts from the format [41] that is established within the context when the mother, regardless of the intention of the child, can give a meaning to his actions because it is only the mind of the other which can found in the autistic child a mental state that makes him a talking of his native language.

In Turtle Project [35] dyadic therapy is privileged just to allow a new form of relationship that helps the child to live the dependence in a new form and to learn new patterns of communication. Unlike what happens in other approaches, the therapist is an active part in the interaction, a living companion [42] acting as a facilitator for communication and as a model to be imitated by parents who do not yet know how to respond and interpret the mainly bodily child's messages. The therapist is there, as Winnicott would say [43] [44], where the child can meet him, and helps the mother-child pair to tolerate the inevitable frustrations that arise from the difficulties in finding an attunement. An attunement which applies only to that child who is in relationship with his mother, and not to a couple in an abstract sense, because every relationship is unique, and each individual is what becomes according to the personal patrimony and in reference to the stimuli received from the environment. Learn to tolerate the inevitable frustrations necessary to find the same significance to the events is what founds the profound sense of language and that allows the learning of meanings shared by the community.

In a general sense the aim of the developmental approach of Turtle Project is to create natural settings in which to support, through a bodily intervention, the developmental trajectories of each child, helping him to integrate the various senses and to meet the body of other people without being afraid of.

In this sense, besides the dyadic therapy, group interventions are proposed to enable the embodied simulation with peers in motivating contexts where the mirroring of dysfunctional behaviors acts as a further stimulus to attune. In the peer group supported by therapists (at least 2 professionals to 4 children) the child find a natural framework in which give shape, that is format [41] contents not yet integrated and where, at least initially, also the dysfunctionality can become an adaptive form if the others can understand or share it. The objectives, calibrated for the single group, are gradually modified to allow more structured communication layers and the expansion of language occurs as the child's need to exchange and not for imposition from outside. In this way, also the modeling that the therapist puts in place to improve the linguistic expression is accepted as 
main driver of the relationship and not as incomprehensible claim for a performance.

Once the child is able to find a good level of attunement with the outside world, it is possible to propose him more structured interventions that support the cognitive development with modalities more advanced than those necessary to be present to the other in an affective relationship.

To demonstrate how the bodily-affective dimension is crucial in the structuring of the autistic disorder and how it takes priority over the cognitive disharmony some studies [45] [46] [47] [48] highlighted, through the evaluation protocol called T.U.L.I.P. ${ }^{1}$, how a sample of 49 children, regardless of the ADOS score [49], showed a better ability to understand intentions and emotions of the others. By monitoring the group during 4 years of Turtle Project therapy [35] there was evidence that the results were significantly_better as early as the end of the second year of therapy, compared with children who had a worse outcome at the T.U.L.I.P. protocol testing. As well as allowing the identification of the zone of proximal development, the T.U.L.I.P. protocol clearly shows that the main obstacle to the development is determined by the lack of those basic attunement mechanisms that are highlighted through the UOI and the TCE [47].

This approach requires, as can be seen from the above reflections, a therapist competence both in the field of the development theories to calibrate the intervention according to the developmental level of the child, both in the relational area in order to contain the anxieties, the frustrations, the attacks on linking [36], the atypical behavioral that dot the intervention with parents too. That is why in Turtle Project approach there are psychotherapists of developmental age and professionals with specialized developmental relationship training.

\section{Conclusions}

This theoretical and clinical framework allows to elaborate also the emerging difficult emotional dynamics. The knowledge of the technique itself is not sufficient to guarantee the capacity to hold child's and own's reactions, because the risk is to put in place aggressive behaviors to evade the sense of impotence felt. The rigidity of some methods colludes with the persistence of the child to protect himself from the outside and amplifies his obsessive behaviors giving rise to an unsuccessful escalation which can be transformed only if profoundly elaborated by the therapist. It is not useful to answer the segmentation with a symmetrical behavior because the primary goal is to encourage a possible integration. On the other hand, the indispensable premise of the intervention is starting from the child and not from the symptoms present, this to ensure, besides the change, also his right to childhood. The starting point is always the body as a place of primordial feelings that need to be gathered, accepted and elaborated from the${ }^{1}$ The T.U.L.I.P. protocol investigates the autism severity through the ADOS, the IQ through the Leiter-R, the ability to understand the intentions of others through the Understanding of Other Intention (UOI) test and the presence of emotional contagion through the Emotional Contagion Test (TCE). 
rapist's mind in order to share and mentalize experiences.

It appears necessary, especially in the early stages of therapy, that the intervention is not fragmented among different operators and disciplines because this would collude with the already existing fragmentation of the child and his context while therapists must act as facilitators in all directions. Multiple operators may work in the Turtle Project approach because they all perform the living companion function. This activates the existing resources by channeling the energies of the child towards the next stage of development without having to distinguish too early the psychomotor intervention from the speech therapy, because the child needs to integrate his knowledges at the time when he lives them, so the words acquire their deep meaning if they arise from the action and if are shared in the play. The ludic dimension, that is to play and do not play games, is what should characterize all interventions so that enjoyment and motivation could activate the cognitive functions. To get involved is what promotes the activation of the ludic area that should never be confused with the use of games for educational purposes but is constituted by shared action that generates pleasure and encourages to look for the other, in order to avoid those self-stimulations which, in absence of other, allow the child to experience few moments of rest. In a research conducted to assess the efficacy of the developmental approach [35], in a sample of 80 autistic children, significant improvements were founds in all developmental areas: significant change was found on Cognitive abilities (F2, 78 $\left.=32.06, \mathrm{p}<0.001 ; \eta^{2}=0.29\right)$; on ADOS score $\left(\mathrm{F} 2,78=65.15 ; \mathrm{p}<0.001, \eta^{2}=\right.$ 0.45 ); on Stereotyped behaviors (Wilks' lambda: 0.76; $p<0.001, \eta^{2}=0.24$ ). In fact, after 4 years of therapy, 31 children (39\%) came out from the ADOS diagnostic category of autism, and 14 children (15\%) changed ADOS diagnosis from autism to spectrum. The other children, although remaining in the same ADOS diagnostic classification, showed a decrease in the ADOS score and consequently also in the autistic symptomatology for all the areas taken into account (communication, reciprocal social interaction, play, restricted and repetitive behaviors). Another significant finding emerged from the research in question, was the increase in IQ score in the most of the children (\%) which showed that lowering the defenses and growing the relational opening, if realized through an intervention that uses the body in the rehabilitative relationship with the child, make it possible a better use of the cognitive potential.

In light of the foregoing, it would be advisable that the bodily dimension was more considered in the treatment of children in the autistic spectrum and that it was given greater importance to the psychomotor area during the assessment and naturally during the therapeutic process.

The future perspective of therapies for children with autism spectrum disorder should therefore be to promote more and more the attunement processes that involve the body as a place of primordial affection and feelings. The interchangeability of sensory modes, activated by the attunement processes, in fact, supports behavioral expressions paving the way for the structuring of cognitive processes. If the child has been able to experience the various sensory elements, 
learning how to adjust depending on interaction with each other, he will have the tools needed for solving the problems that world experience requires. Cognitive maturity, as Bruner points out [50], depends on the relationship of enactive, iconic and symbolic systems of representation.

\section{Compliance with Ethical Standards}

All procedures performed in studies involving human participants were in accordance with the ethical standards of the institutional and/or national research committee and with the 1964 Helsinki declaration and its later amendments or comparable ethical standards.

\section{Informed Consent}

Informed consent was obtained from all individual participants included in our previous studies and citated in this paper.

\section{References}

[1] American Psychiatric Association (2013) Diagnostic and Statistical Manual of Mental Disorders (DSM-5). Washington DC.

[2] Vygotskjy, L.S. (1934) Thought and Language. MIT Press, Cambridge.

[3] Trevarthen, C. (1977) Descriptive Analyses of Infant Communicative Behavior. In: Schaffer, H.R., Ed., Studies in Mother-Infant Interaction, Academic Press, London, 227-270.

[4] Trevarthen, C. (1998) The Concept and Foundations of Infant Intersubjectivity. In: Braten, S., Ed., Intersubjective Communication and Emotion in Early Ontogeny, University Press, Cambridge, 15-46.

[5] Trevarthen, C. (2001) Infant Intersubjectivity: Research, Theory, and Clinical Applications. Journal of Child Psychology and Psychiatry, 42, 3-48. https://doi.org/10.1111/1469-7610.00701

[6] Schore, A. (2003) Affect Dysregulation and Disorders of the Self. Norton, New York.

[7] Schore, A. (2012) The Science of the Art of Psychotherapy. Norton, New York.

[8] Tronick, E. (2007) The Neurobehavioral and Social-Emotional Development of Infants and Children. Norton, New York.

[9] Stern, D. (1985) The Interpersonal World of the Infant. Basic Books, New York.

[10] Stern, D. (1995) The Motherhood Constellation: A Unified View of Parent-Infant Psychotherapy. Basic Books, New York.

[11] Stern, D. (2004) The Present Moment in Psychotherapy and Everyday Life. Norton, New York.

[12] Nadel, J. (2014) How Imitation Boosts Development in Infancy and Autism Spectrum Disorder. Oxford University Press, Oxford.

[13] Reddy, V. (2008) How Infants Know Minds. Harvard University Press, Cambridge.

[14] Eisenberg, N. (1995) Social Development. Sage Publications, Thousand Oaks.

[15] Feldman, R. (2007) Parent-Infant Synchrony and the Construction of Shared Timing: Physiological Precursors, Developmental Outcomes, and Risk Conditions. Journal of Child Psychology and Psychiatry, 48, 329-354. 
https://doi.org/10.1111/j.1469-7610.2006.01701.x

[16] Tustin, F. (1972) Autism and Childhood Psychosis. Hogarth, London.

[17] Tustin, F. (1981) Autistic States in Children. Routledge, London.

[18] Tustin, F. (1986) Autistic Barriers in Neurotic Patients. Karnac Books, London.

[19] Tustin, F. (1990) The Protective Shell in Children and Adults. Karnac Books, London.

[20] Tustin, F. (2013). Autistic States in Children. Revised Edition, Routledge, London.

[21] Smith, A. (2009) The Empathy Imbalance Hypothesis of Autism: A Theoretical Approach to Cognitive and Emotional Empathy in Autistic Development. The Psychological Record, 59, 489-510. https://doi.org/10.1007/BF03395675

[22] Meltzer, D. (1975) Explorations in Autism: A Psychoanalytical Study. Clunie Press, Perthshire.

[23] Fordham, M. (1976) The Self and Autism. Medical Books, London.

[24] Damasio, A. and Carvalho, G.B. (2013) The Nature of Feelings: Evolutionary and Neurobiological Origins. Nature Reviews. Neuroscience, 14, 143-152.

[25] Damasio, A. (2010) Self Comes to Mind: Constructing the Conscious Brain. Pantheon Books, New York.

[26] Gaddini, E. (1969) On Imitation. International Journal of Psycho-Analysis, 50, 475-484.

[27] Winnicott, D.W. (1965) The Family and Individual Development. Tavistock, London,

[28] Winnicott, D.W. (1965) Maturational Processes and the Facilitating Environment: Studies in the Theory of Emotional Development. Hogarth Press, London.

[29] Winnicott, D.W. (1974) Fear of Breakdown. International Review of Psychoanalysis, 1, 103-107.

[30] Wallon, G. (1947) Les origines de la penseè chez l'enfant. [The Origins of Thinking in Children.] Presses Universitairesde France, Paris.

[31] Gallese, V. (2001) The "Shared Manifold" Hypothesis: from Mirror Neurons to Empathy. Journal of Consciousness Studies, 8, 33-50.

[32] Gallese, V. (2003) The Roots of Empathy: The Shared Manifold Hypothesis and the Neural Basis of Intersubjectivity. Psychopathology, 4, 24-47. https://doi.org/10.1007/BF03395675

[33] Gallese, V. and Lakoff, G. (2005) The Brain's Concepts: The Role of the Sensory-Motor System in Conceptual Knowledge. Cognitive Neuropsychology, 22, 455-479. https://doi.org/10.1080/02643290442000310

[34] Hobson, R.P. and Lee, A. (1999) Imitation and Identification in Autism. Journal of Child Psychology and Psychiatry, 40, 649-659.

https://doi.org/10.1111/1469-7610.00481

[35] Di Renzo, M., Bianchi di Castelbianco, F., Vanadia, E., Petrillo, M., Racinaro, L. and Rea, M. (2016) From the Emotional Integration to the Cognitive Construction: The Developmental Approach of Turtle Project in Children with Autism Spectrum Disorder. Autism Open Access, 6, 160.

[36] Bion, W.R. (1962) Learning from Experience. William Heinemann, London.

[37] Bion, W.R. (1967) Second thOughts. William Heinemann, London.

[38] Bruner, J.S. (1973) The Relevance of Education. Norton, New York.

[39] Bruner, J.S. (1991) Self-Making and World-Making. Journal of Aesthetic Education, 
25, 67-78. https://doi.org/10.2307/3333092

[40] Bruner, J.S. (1999) The Intentionality of Referring. In: Zelazo, P.D., Astington, J.W. and Olson, D., Eds., Developing Theories of Intention: Social Understanding and Self-Control, Mahwah, Erlbaum, 329-339.

[41] Bruner, J.S. (1983) Child's Talk: Learning to Use Language. Norton, New York.

[42] Alvarez, A. (2012) The Thinking Heart: Three Levels of Psychoanalytic Therapy with Disturbed Children. Routledge, Hove.

[43] Winnicott, D.W. (1971) Playing and Reality. Tavistock, London.

[44] Winnicott, D.W. (1989) Psychoanalytic Explorations. Karnac Books, London.

[45] Di Renzo, M., Bianchi di Castelbianco, F., Petrillo, M., Racinaro, L. and Rea, M. (2015) Assessment of a Long-Term Developmental Relationship-Based Approach in Children with Autism Spectrum Disorder. Psychological Reports, 117, 26-49. https://doi.org/10.2466/15.10.PR0.117c15z8

[46] Di Renzo, M., Bianchi di Castelbianco, F., Vanadia, E., Petrillo, M., Racinaro, L. and Rea, M. (2016) Assessment of Executive Functions in Preschool-Aged Children with Autism Spectrum Disorders: Usefulness and Limitation of BRIEF-P in Clinical Practice. Journal of Child and Adolescent Behavior, 4, 313. https://doi.org/10.4172/2375-4494.1000313

[47] Di Renzo, M., Bianchi di Castelbianco, F., Vanadia, E., Petrillo, M., Racinaro, L. and Rea, M. (2016) T.U.L.I.P. Protocol (TCE, UOI, Leiter-R as Indicators of Predictivity) for the Assessment of the Developmental Potential in Children with Autism Spectrum Disorders. Autism Open Access, 6, 188. https://doi.org/10.4172/2165-7890.1000188

[48] Di Renzo, M., Bianchi Di Castelbianco, F., Petrillo, M., Racinaro, L., Donaera, F. and Rea, M. (2016) The Emotional Contagion in Children with Autism Spectrum Disorder. Austin Journal Autism \& Related Disability, 2, 1020.

[49] Lord C., Rutter M., Di Lavore P.C. and Risi S. (2005). ADOS-Autism Diagnostic Observation Schedule. O.S. Organizzazioni Speciali, Firenze.

[50] Bruner, J.S. (1966) Toward a Theory of Instruction. Harvard University Press, Cambridge, Mass.

\section{Abbreviation Note List}

Pervasive Developmental Disorders (PDDs)

Diagnostic and Statistical Manual of Mental Disorders, 5th edition (DSM-5)

Restricted and Repetitive Behaviors (RRB)

Emotional Contagion Test (TCE)

Understanding of Other Intention (UOI)

Leiter International Performance Scale-Revised (LEITER-R)

Autism Diagnostic Observation Schedule (ADOS)

TCE, UOI, Leiter-R as Indicators of Predictivity (T.U.L.I.P.) 